\title{
On the Causes and Countermeasures of the Psychological Problems of "Good Children" from Their Own Perspective
}

\author{
$\mathrm{Hu} \mathrm{Yan}^{1, \mathrm{a}}$
}

${ }^{1}$ Foundation Department of Chongqing Real Estate Vocational College, College Station, Chongqing, China a295738740@qq.com

\begin{abstract}
Through the discussion of the individual self-reasons for the psychological problems of "good children" , it is helpful for "good children" to better understand themselves, know themselves and accept themselves, so as to promote "good children" self-education in real life, consciously Change Self, truly become independent personality, physical and mental health.
\end{abstract}

Keywords: "good children", psychological problems

\section{从自身角度探讨“乘孩子”心理问题成因和对策 \\ 胡妍 $1, \mathrm{a}$}

\section{1 重庆房地产职业学院基础系 大学城 沙坪坝区 重庆 中国 \\ a295738740@qq.com}

\section{摘要}

通过对 “乘孩子” 心理问题形成的个体自身原因的探讨，有利于 “乘孩子” 更好地了解自己，认识自 己，接纳自己，从而促进 “乘孩子” 在现实生活中自我教育，自觉改变自我，真正成为人格独立、身 心健康的人。

\section{关键词: “乘孩子” 心理问题}

\section{1. 引言}

本人在从事个体心理咨询中遇到很多被医院诊断 为抑郁症、焦虑症、强迫症等的孩子, 这些孩子的父母 认为孩子在小时候很乘, 成绩也不错, 各方面都表现优 秀, 但是到初中或高中就突然出现了问题, 比如成绩下 降、厌学、和同学交往出现矛盾冲突、被孤立、不愿和 同学说话, 沉迷于网络游戏, 甚至有的孩子出现自残、 自杀行为。从孩子自身角度来看, 这部分 “乘孩子” 的 心理问题形成原因有哪些方面?

\section{2. “靟孩子” 心理问题形成的个体原因}

\section{1. “乘” 是一种人格面具}

“乘” 是 “乘孩子” 的一种人格面具, 人格面具是 指个体在社会生活中, 为了适应社会, 而扮演不同的社 会角色, 这样的 “我” , 并非真实的 “我”, 而只是一
种人格面具。我国的教育过于偏重于社会价值取向，轻 视孩子的心理需求。导致我们的“乘孩子” 会放弃个体 的内在需求, 而去迎合社会的要求。很多家长认为孩子 就像是一块白板, 大人可以按照自己的意愿在上面画出 任何美妙的风景, 而实际上每个孩子都有自己的精神胚 胎, 孩子的成长发展都建立在这个精神胚胎的基础上, 而不能按照家长的意愿随心所欲地发展。我们的家长给 孩子设立了很多的条条框框，如：“大人讲话，小孩莫 插嘴”，“小孩不懂别瞎问”，以及通过赞许鼓励某些 行为来规范孩子, 如: “真乘”, “真听话” , 企图规 制出一个个父母眼中 “乘的好孩子”。文化、社会、家 庭、学校给孩子安排了很多 “好的模板”，可是，很多 这些模板与孩子的精神胚胎并不相符, 不符合孩子健康 成长的需要, 但是 “乘孩子” 为了获得奖赏而接受了外 在的模板和要求, 就像戴着一副没有弹性的面具生活, 限制了他们内心的自由, 也限制了他们的身心健康成 长。为了 “乘孩子” 的面具, 生活在外在的标准中, 他 们丧失了独立判断能力、丧失了批判精神和勇气、丧失 了自我欣赏的能力, 丧失了创新精神和能力。

\section{2. “乘” 的背后可能是很深的绝望和恐惧}

“乘孩子”, 往往不敢提要求, 不敢发出自己内心 的声音。然而一个健康的有活力的孩子, 必然会发出他 的高兴与不高兴的声音, 提出他合理或不合理的要求。 
的意义，他们往往缺乏激情，没有生命力，他们是没有 自我的。有些 “乘孩子” 会变成两面派的孩子, 他们在 父母、老师、亲戚朋友面前会表现得很乘, 很有礼貌, 很懂事、遵守规则，但是在外面背着父母时可能会欺负 比自己小的孩子，会虐待小动物等。比如有一个初一的 男生, 一直表现很乘巧, 但是在母亲生下妹妹后的第三 天, 他把自己养的小仓鼠从二十楼扔到了楼下, 然后跑 到楼下去看小仓鼠血肉模糊的样子, 在咨询中他表达出 很不想要妹妹, 甚至想把妹妹扔到楼下的想法, 但是他 不敢做, 也害怕父母知道他内心的真实想法, 表面上装 着很喜欢妹妹的样子。两面派型的 “我孩子” 为了达到 自己的目的, 会根据别人的需要和喜好, 在不同场合刻 意地表现出好的言行以迎合别人的需要, 但这些言行往 往是虚假的、伪装的。这种孩子很可能成为伪君子, 甚 至形成分裂的人格。这样的 “乘孩子” 在人际交往中不 敢展示真实的自己，他们会在朋友面前表现得热情、主 动, 乐于助人, 似乎朋友很多, 但却没有一个朋友可以 真正地靠近他们的内心，长期以往便会出现人际交往问 题。

\section{5. 头脑中的“应该”限制了“乘孩子”}

在 “乘孩子” 的头脑中有着很多的 “应该” , 如: “你应该要优秀, 你应该要懂事, 你应该要听话”, 然 而 “靟孩子” 有 “优秀、懂事、听话、勤奋” 的一面, 就一定有 “不优秀、不懂事、不听话、不勤奋” 的另一 面, 我们生活在一个二元对立的世界, 作为一个完整的 人, 必然同时具有这两个面, 不可能只有一面而没有另 外一面。“那么乘孩子 “不优秀、不懂事、不听话” 的一 面一定会被否定和压抑。这些被压抑下去的 “不乘的部 分”, 并没有消失, 它们不时会浮出水面, 造成 “乘孩 子” 的困扰, 但是 “乘孩子” 很害怕去看到它们, 那么 怎么办? 于是 “乘孩子” 会发展出很多策略来逃避这些 “不乘的部分”，以及脑海里面不断冒出来的 “你不够 好、你是错的、你不如别人” 的紧䈨咒, 所以他们会拼 命努力, 拼命去让自己 “优秀、懂事、听话、勤奋”, 但是当他们发现不管他们如何努力, 都无法做到让别人 满意时, 他们开始用酗酒、抽烟、旷课、打游戏等方式 来逃避。

面对以上“乘孩子”心理问题形成的个体自身因素， 我们可以从以下几个方面进行干预，帮助有心理问题的 “乘孩子” 形成自我教育的对策。

\section{3. 自我教育对策}

\section{1. 形成正确的自我意识}

自我意识是个体对自己, 以及自己与外界关系的认 习、生活, 看似一帆风顺, 却可能觉得自己是为父母而 活, 从来没有为自己活过。这样的 “乘孩子” 不知道自 己想要什么, 不知道自己的感受, 不知道自己所做事情

\footnotetext{
1 学会与自己对话 黄其明;- 《上海教育》-2012-06-20
} 
越来越压抑, 越来越不快乐, 因为他们不知道自己是谁, 不知道自己活着的意义。人本主义心理学大师罗杰斯认 为: 当一个人忽视或扭曲自己的内心感受时就可能出现 心理问题。当我们过度在乎他人的看法时, 就会对他人 的反应特别敏感, 从而丧失了自由, 自我的力量也被减 弱。

存在主义心理学认为生命的意义在于选择, “我选 择, 我自由, 我存在”。也就是说当我按照自己的意愿 做了选择，我就会体验到一种自由感，这个时候我就会 有存在的感觉。当 “乘孩子” 可以为自己的一切进行选 择时, 他便是在做自己, 便是成为自己。为自己的一切 进行选择, 意味着 “乘孩子” 需要尊重自己内在的感觉, 需要有自己的立场, 需要倾听自己内在的声音, 然后根 据自己内在的感觉、立场、声音做出自己的判断和选择。 这时 “乘孩子” 的选择就是自己的选择, 而不是别人帮 他做出的选择，他便在做他自己。“乘孩子”之所以缺 少创造力, 习惯于模仿, 原因就是因为 “乘孩子” 很难 做到尊重自己的感觉，习惯于顺从别人的意志。当 “乘 孩子” 可以尊重自己的感觉，相信自己内在的声音，让 生命力在自己身上流淌的时候, 那么这个时候所谓的创 造力就自然而然的就出来了。

“成为自己” 意味着我选择, 我自由, 但也意味着我需 要为我的选择负责任, 结果由我自己来承担, 而不是怪 罪父母、老师等其他人, 让别人来承担后果, 而自己扮 演受害者的角色。“做自己”，按照自己的意愿去生活， 需要的是 “我选择, 我负全责。” “乘孩子” 通过了解 自己，认识自己，接纳自己，真正成为人格独立、身心 健康的人。

\section{REFERENCES}

[1]Hu Yan. (2018) A study on the common psychological problems of"good children". Mental health education in primary and secondary schools, 9:245-247

[2]Zong Rui. (2008) Whether a good boy is a good boy or not. China Health nutrition-lohas, 4:118-119.

[3]Yan Yi.(2012) The back of left-behind children's "being well-behaved" —— How do parents cope with children's mental health. Healthy Living, $2: 32-33$

[4]Wang Qian.,Chen Huichang.(2007) The relationship between parental monitoring and adolescents 'problem behaviors and its implications for intervention. Educational theory and practice, 10:35-37.

[5]Sheng Jiahui. (2016) Is it really a good thing for children to be obedient. Mother and child healthy, 10:70-71. 